\title{
Artefactos Líticos en la Hacienda Boulder, Municipio de Palermo , Departamento del Huila
}


Plancha 1. Mapa de localización de los sitios. Boulder 1 $-2-3-4$

Lámina 1.

Aspecto de los sitios arqueológicos y paleontológicos, de la Hacienda Boulder

Lámina 2.

Raspadores triangulares

Lámina 3.

Lascas triangulares retocadas

Lámina 4.

Raspadores cóncavos

Lámina 5.

Raspadores laterales

Lámina 6.

Raspadores discoidales

Lámina 7.

Raspadores ovoides

Lámina 8.

Lascas rectangulares retocadas

Lámina 9.

Golpeadores nucleares 
La exploración materia de este informe se efectuó teniendo en cuenta la información suministrada por el Dr. Enrique Díaz C., sobre el hallazgo de un molar fósil en terrenos de la hacienda Boulder, situada en las proximidades de Neiva. La pieza dentaria procedente de la localidad corresponde a un mastodonte; basados en este hecho, las condiciones geográficas de la región, y el antecedente registrado en el municipio de Garzón (Depto. del Huila), en donde anteriormente se constataron evidencias fósiles de megafauna (megaterio y mastodonte) junto con algunos xilópalos fragmentados y aparentemente tallados (H. Burgl 1958) cuya estratificación está claramente establecida (Van-der-Hammen,1958) efectuamos este reconocimiento con la finalidad de localizar algunos sitios paleo-indios.

\section{DESCRIPCION DEL AREA DE ESTUDIO}

La hacienda Boulder está situada a una distancia de $5 \mathrm{~km}$. de la ciudad de Neiva y a $56{ }^{\circ} \mathrm{NW}$ con relación a esta ciudad; forma parte del municipio de Palermo. Al lugar se llega por la vía principal que une la capital del Huila con la ciudad de Bogotá.

La altura sobre el nivel del mar en los terrenos de la hacienda fluctúa entre 432 y $500 \mathrm{~m}$.

Los terrenos, en general muy erosionados, corresponden en su mayor parte a terrazas con las que alternan colinas muy bajas. Algunas praderas cubren la hacienda actualmente destinada a la ganadería, pero tambien gran parte de la vegetación corresponde al bosque muy seco tropical (Formaciones vegetales de Colombia, 1963). Esta formación tiene como límites climáticos una temperatura media superior a 240 centígrados y un promedio de lluvia entre $\operatorname{los} 500$ y $1.000 \mathrm{~mm}$., es característica del río Magdalena, por las regiones de Aipé, Neiva y Villavieja.

Geológicamente se clasifican los terreros dentro de la formación Honda (mapa geológico plancha No. 8 Neiva) con areniscas fluvio-lacustres, conglomerados y arcillas de color pardo-rojizo. La formación correspondiente a la "terraza de Aipé", es observable en varios sitios de Boulder, y la edad asignada a esta formación, basada en datos flúor-cronológicos corresponde a la parte superior del penúltimo glacial (Riss) y parte inferior del último interglacial (Riss-Würm) (1)

(1) Información personal Dr. Thomas Van-der-Hammen 198

\section{LOS SITIOS ARQUEOLOGICOS}

A los sitios arqueológicos se llega por un carreteable que atraviesa la hacienda, para finalmente llegar a las localidades de Llano del Sen y La Soledad; este carreteable se desprende de la via principal a Bogotá en dirección oeste.

Para la demarcación de los cuatro sitios referidos en este informe, se tomó como punto de referencia un kiosko para suministro de sal a los ganados de la hacienda, que está situado a $1.5 \mathrm{Km}$. W. de la entrada al carreteable de penetración.

\section{Sitio 1 (Boulder 1) (Plancha 1, lámina 1.figs. 1-2)}

Se localiza en las proximidades al punto de referencia; después de efectuarse una recolección superficial, se procedió a la demarcación de un corte de observación de $2 \mathrm{x}$ $2 \mathrm{~m}$.. dividiéndose éste para efectos de control, en cuatro cuadrículas de $1 \times 1 \mathrm{~m}$.

Este corte se efectuó a una distancia de 74 m.N. -33 W. con relación al punto de referencia. Se utilizaron niveles estratigráficos arbitrarios de $0.10 \mathrm{~cm}$. en razón de que aún no conocíamos la estratigrafía física del sitio. La escogencia de este lugar para corte, se efectuó teniendo en cuenta la concentración superficial de material lítico.

En los $7 \mathrm{~cm}$. superiores (nivel 1) de la cuadrícula (75N.-33W.) (capa vegetal-tierra gris algo parduzca) se localizaron 2 fragmentos de Chert, material minerógeno básico en la elaboración de los implementos líticos del lugar, pero ninguno de estos fragmentos presenta huellas de utilización o modificaciones intencionales.

$\mathrm{Ni}$ en este nivel ni en los subsiguientemente excavados se obtuvo material orgánico oseo ni muestras de carbón; esta cuadrícula se profundizó hasta $1.70 \mathrm{~m}$. sin resultados positivos; las siguientes cuadrículas excavadas (74N.-35W.) $(74 \mathrm{~N} .-36 \mathrm{~W})(75 \mathrm{~N} .-36 \mathrm{~W}$ ) resultaron completamente estériles a partir de la superficie.

Un perfil estratigráfico sobre la pared oriental del corte da la siguiente secuencia:

$0-12 \mathrm{~cm}$. Capa vegetal (tierra gris algo parduzca)

$12-15 \mathrm{~cm}$. Arcilla parduzca

15 y más cm. Arcilla gris muy compacta con abuntantes inclusiones de arenisca.

El sitio 1, presenta la mayor concentración de elementos líticos superficiales, especialmente en el lugar donde efectuamos el corte y en los alrededores del área de aproximadamente $50 \mathrm{~m}^{2}$.

En la recolección superficial se obtuvieron 344 elementos líticos con la siguiente distribución:

Lascas simples

Núcleos fragmentados sin huellas de utilización

Lascas retocadas

Raspadores circulares

Raspadores triangulares

Raspadores laterales

Núcleos desbastados

Raspadores cóncavos

Raspadores terminales 
Fragmento de golpeador Golpeador completo ovoide TOTAL

Sitio 2 (Boulder 2) (Plancha 1, lámina 1, fig.3)

Esta localizado a una distancia de 250 m.S.E. con relación al punto de referencia este sitio está localizado sobre las laderas muy erosionadas que bordean una depresión o jagüey donde durante el invierno se acumulan las aguas. Hacia el extremo occidenta de la depresión, en el fondo de la misma, se localizó el molar de Haplomastodón al que aludimos anteriormente; sin embargo, a pesar de minuciosa inspección, no aparecieron otros fragmentos osteológicos en las barrancas erosionadas; este problema solamente puede ser esclarecido mediante posteriores excavaciones de mayor amplitud.

En la inspección verificada en este sitio, se obtuvieron 22 elementos líticos dispersos sobre las laderas erosionadas en una superficie de aproximadamente $120 \mathrm{~m}^{2}$, la distribución de tales elementos es la siguiente:

Fragmentos de Chert (lascas) sin ninguna evidencia de utilización Fragmento de núcleo con señales de utilización en uno de los bordes de fractura Fragmento de núcleo oval

\section{TOTAL

Estos objetos líticos aparecieron superficiales; el hecho de no encontrar evidencia líticas en los estratos visibles, en las barrancas erosionadas, nos hace pensar que estos elementos descendieron de la parte plana superior al fondo, por efectos de la erosión por tanto es imposible establecer alguna correlación entre el elemento cultural y el molar fósil allí encontrados y menos aún tratar de plantear posibles consideraciones de orden cronológico basados en esta situación.

Una observación vertical de las barracas erosionadas que bordean la depresión permite establecer la siguiente secuencia estratigráfica:

$0-0,49 \mathrm{~cm}$. capa vegetal; tierra gris algo parduzca con inclusiones cuarcíticas $0,49-225 \mathrm{~cm}$. arcilla gris compacta con abundantes inclusiones de arenisca.

225 y más $\mathrm{cm}$. arcilla parduzca rojiza compacta.

Sitio 3 (Boulder 3) (Plancha 1, lámina 1, fig. 4)

Está ubicado a $450 \mathrm{~m}$. aproximadamente, con relación al punto de referencia; corresponde a una superficie plana de $250 \mathrm{~m}^{2}$. con ligeras ondulaciones del terreno sobre la superficie aparecen dispersos los elementos líticos. La mayor parte del material recolectado corresponde a elementos obtenidos mediante percusión, sin embargo, 3 de los elementos recolectados ( 2 fragmentos de metate, 1 fragmento de mano de moler) son pulimentados; la distribución establecida sobre 64 elementos obtenidos es la siguiente:
Raspadores circulares

Lascas sin modificar

Lascas retocadas

Núcleos fragmentados

Raspadores laterales

Raspadores cóncavos

Raspadores triangulares

Golpeador (chopper)

Fragmentos de metate

Fragmento de mano de moler

TOTAL

Sitio 4 (Boulder 4) (Plancha 1, lámina 1, fig.5)

A una distancia de $550 \mathrm{~m}$. en dirección sur con relación al punto de referencia se localiza el sitio, el cual corresponde también a una depresión (jagüey). Su importancia radica en que sobre las laderas erosionadas de mediana inclinación (450) aparecen abundantes elementos óseos silicificados, algunos de los cuales están en identificación. No pudo establecerse asociación de elementos líticos con los huesos en mención, y solamente 3 fragmentos de núcleo (chert) sin ninguna evidencia de elaboración humana se obtuvieron en el fondo de la depresión hacia el extremo norte. Los elementos óseos se encuentran in situ, en el estrato correspondiente a arcilla gris compacta con abundantes inclusiones de arenisca; la estratigrafía es muy similar a la del sitio 1 ; es probable que los fragmentos de núcleo encontrados, hayan descendido de la superficie plana superior hacia el fondo, a causa de la erosión del terreno.

\section{DESCRIPCION DE ALGUNOS DE LOS IMPLEMENTOS}

A continuación presentamos la descripción de algunos de los implementos de Boulder, sin pretender establecer tipologías, teniendo en cuenta las bajas frecuencias del material estudiado, aunque se observan tendencias en las diferentes formas que a continuación se describen:

\section{Raspadores Triangulares}

A) Sitio: Boulder 1 (Lámina 2, figs. A-A')

\section{Material: (chert)}

\section{Dimensiones:}

longitud: $47 \mathrm{~mm}$.

anchura: $44 \mathrm{~mm}$.

espesor en la base: $18 \mathrm{~mm}$.

espesor en el extremo: $2 \mathrm{~mm}$

Los bordes convergen hacia el extremo agudo; uno de los bordes es aserrado en toda su longitud, el otro extremo sólo presenta aserramiento hacia el extremo distal. Sobre las superficies, junto a los bordes se observan pequeños retoques a presión. 
B) Sitio: Boulder 1(Lámina 2, figs. B-B')

\section{Material: (chert)}

Dimensiones:

longitud:52 $\mathrm{mm}$.

anchura basal: $44 \mathrm{~mm}$.

espesor medio: $15 \mathrm{~mm}$

espesor mínimo: $7 \mathrm{~mm}$.

Uno de los bordes del artefacto presenta denticulaciones; la superficie anterior está desbastada y la posterior es lisa y plana.

C) Sitio: Boulder 1 (Lámina 2, figs. C-C')

Material: (chert)

Dimensiones:

longitud: $53 \mathrm{~mm}$.

anchura: $41 \mathrm{~mm}$.

espesor basal: $21 \mathrm{~mm}$.

espesor en el extremo agudo: $2 \mathrm{~mm}$.

Bordes muy convergentes, la superficie anterior hacia el borde izquierdo y el extremo proximal presenta retoques por presión.

D) Sitio: Boulder 1 (Lámina 2, figs. D-D')

Material: (chert)

Dimensiones:

longitud: $46 \mathrm{~mm}$.

anchura: $50 \mathrm{~mm}$.

espesor medio: $12 \mathrm{~mm}$

La superficie anterior del artefacto presenta desbastamientos hacia los bordes y la base: en el plano posterior o de percusión hay retoques secundarios por presión.

\section{Lascas Retocadas Tringulares}

A) Sitio : Boulder 3 (Lámina 3. figs. A-A')

\section{Mąterial: (chert)}

Dimensiones:

longitud: $43 \mathrm{~mm}$.

anchura: $34 \mathrm{~mm}$

espesor medio: $13 \mathrm{~mm}$

La superficie anterior presenta desbastamientos hacia los bordes y base; hacia el extremo distal se observa la corteza original; retoques secundarios por presión son visibles hacia los bordes convergentes. La superficie posterior corresponde al plano de percusión.
B) Sitio: Boulder 1 (Lámina 3, fig. B)

\section{Material: (chert)}

Dimensiones:

longitud: $43 \mathrm{~mm}$.

anchura: $39 \mathrm{~mm}$

espesor basal máximo : $9 \mathrm{~mm}$.

espesor en el extremo agudo: $2 \mathrm{~mm}$.

El implemento presenta adelgazamiento hacia los bordes, y pequeños retoques por presión. En la base aparece la corteza original.

C) Sitio: Boulder 1 (Lámina 3, figs. C-C')

\section{Material: (chert)}

Dimensiones:

longitud: $41 \mathrm{~mm}$.

anchura: $34 \mathrm{~mm}$

espesor: $10 \mathrm{~mm}$.

En la lasca, tanto los bordes laterales convergentes como el borde basal son aserrados y el espesor disminuye hacia estos; observándose retoques a presión; la superficie anterior está desbastada y la posterior corresponde al plano de percusión.

D) Sitio: Boulder 1 (Ĺámina 3, figs. D-D’)

\section{Material: (chert)}

Dimensiones:

longitud: $36 \mathrm{~mm}$

anchura: $31 \mathrm{~mm}$.

espesor máximo: $9 \mathrm{~mm}$.

Hacia los bordes se observan retoques secundarios: tanto los bordes laterales como el de la base están aserrados.

\section{Raspadores Cóncavos}

A) Sitio: Boulder 3 (Lámina 4, figs. A-A’)

\section{Material: (chert)}

Dimensiones:

longitud: $71,5 \mathrm{~mm}$.

anchura máxima: $42 \mathrm{~mm}$

espesor medio: $9 \mathrm{~mm}$.

El artefacto presenta un adelgazamiento en forma de bisel hacia su borde de utilización cóncavo y retoques por presión hacia ésta; en el borde opuesto a la concavidad se evidencian huellas de utilización. 
B) Sitio: Boulder 1 (Lámina 4, figs. B-B')

Material: (chert)

\section{Dimensiones:}

longitud: $55.5 \mathrm{~mm}$.

anchura máxima: $32 \mathrm{~mm}$.

espesor máximo: $12 \mathrm{~mm}$.

El borde aserrado marginal de utilización presenta concavidad entre la parte media y el extremo agudo del artefacto. En conjunto, el implemento presenta desbastamientos y retoques por presión, con mayor intensidad en la porción cóncava. En el lado opuesto al borde longitudinal de utilización aparece la corteza original.

C) Sitio: Boulder 3 (Lámina 4, fgs. C-C')

\section{Material (chert)}

\section{Dimensiones:}

longitud: $51 \mathrm{~mm}$.

anchura máxima: $43 \mathrm{~mm}$

espesor medio: $14 \mathrm{~mm}$.

Hacia el borde de utilización cóncavo, el implemento está desbastado y retocado mediante presión, este borde es aserrado y cortante. La superficie posterior corresponde al plano de percusión.

D) Sitio: Boulder 1 (Lámina 4, figs.D-D')

\section{Material: (chert)}

\section{Dimensiones:}

longitud: $59 \mathrm{~mm}$.

anchura: $38 \mathrm{~mm}$.

espesor máximo: $22.5 \mathrm{~mm}$.

Hacia el borde longitudinal de utilización la superficie opuesta al plano de percusión, presenta desbastamientos; el borde, presenta doble concavidad. Se observa una porción de la capa cortical adherida.

\section{Raspadores Laterales.}

A) Sitio: Boulder 1 (Lámina 5, figs. A-A')

Material: (chert)

Dimensiones:

longitud: $62 \mathrm{~mm}$

anchura media: $26 \mathrm{~mm}$

espesor: $13.5 \mathrm{~mm}$.

La superficie dorsal, hacia el borde de utilización muestra retoques secundarios por presión. La superficie está constituída por la capa cortical adherida.
B) Sitio: Boulder 1 (Lámina 5, figs. B-B')

\section{Material: (chert)}

\section{Dimensiones:}

longitud: $45 \mathrm{~mm}$.

anchura: $33.5 \mathrm{~mm}$

grosor en el lado opuesto al borde: $21 \mathrm{~mm}$.

El artefacto presenta adelgazamiento hacia el borde de utilización. Esta forma se logra por percusión; hacia el borde sinuoso de utilización hay retoques secundarios por presión. Los planos laterales y el opuesto al borde de utilización son verticales; el plano posterior corresponde a la corteza original.

C) Sitio: Boulder 1 (Lámina 5, figs. C-C')

\section{Material: (chert)}

Dimensiones:

longitud:57 $\mathrm{mm}$.

anchura máxima: $45 \mathrm{~mm}$.

espesor máximo: $20 \mathrm{~mm}$.

La superficie dorsal está desbastada hacia el borde de utilización y junto a este aparecen retoques secundarios. En el lado opuesto al borde de utilización sobre la corteza original también aparecen algunos retoques por presión. La superficie posterior corresponde al plano de percusión.

D) Sitio: Boulder 1 (Lámina 5, figs. D-D')

\section{Material: (chert)}

Dimensiones:

longitud: $72 \mathrm{~mm}$.

anchura máxima: $29 \mathrm{~mm}$.

espesor máximo: $26 \mathrm{~mm}$.

Para la elaboración del artefacto se utilizó un fragmento de núcleo alargado. La mayor parte del contorno superficial corresponde a la corteza original. Sobre la superficie de fractura, dos desbastamientos se produjeron hacia el borde de utilización, el cual presenta contorno ondulado; los retoques secundarios hacia este borde determinan aserramiento.

\section{Raspadores Discoidales.}

Bajo esta denominación agrupamos aquellos implementos que presentan más de dos bordes de utilización o forma más o menos circular con bordes de utilización periféricos. Los contornos varían de circular a oval y oblongo, como puede observarse en las planchas (véanse planchas) in embargo, no denominamos subtipos, dada la baja frecuencia de implementos que disponemos para este trabajo. 
A) Sitio: Boulder 3 (Lámina 6, figs. A-A')

\section{Material: (chert)}

Dimensiones:

diámetro mayor: $51 \mathrm{~mm}$.

diámetro menor: $46.5 \mathrm{~mm}$.

espesor máximo: $22.5 \mathrm{~mm}$.

El implemento presenta retoques secundarios por presión en la periferia y borde marginal de contorno aserrado en toda su longitud, excepto en uno de los lados; el borde de relieve en conjunto es sinuoso.

B) Sitio: Boulder 1 (Lámina 6, figs. B-B’)

\section{Material: (chert)}

\section{Dimensiones:}

diámetro mayor: $46 \mathrm{~mm}$.

diámetro menor: $34 \mathrm{~mm}$.

espesor máximo: $22.5 \mathrm{~mm}$.

El artefacto presenta desbastamientos en su superficie convexa que determinan 3 bordes marginales, junto a los cuales se ven retoques secundarios por presión; los bordes son de contorno ligeramente convexo y sobre el lado opuesto al borde anterior se observa la corteza original. La superficie opuesta a la convejidad, corresponde al plano de percusión.

C) Sitio: Boulder 1 (Lámina 6, figs. C-C')

\section{Material: (chert)}

Dimensiones:

diámetro mayor : $33.3 \mathrm{~mm}$

diámetro menor: $30.0 \mathrm{~mm}$.

espesor central: $18.0 \mathrm{~mm}$.

La superficie convexa del artefacto está desbastada del centro hacia la periferia y conserva una porción de la corteza original en todo el borde de contorno, se observan retoques secundarios a presión.

D) Sitio: Boulder 1 (Lámina 6, figs. D-D')

\section{Material: (chert)}

\section{Dimensiones:}

diámetro mayor: $55 \mathrm{~mm}$.

diámetro menor: $48 \mathrm{~mm}$.

espesor medio: $10 \mathrm{~mm}$.

Una de las superficies del instrumento de lasca está constituído por la corteza original, la otra corresponde al plano de percusión; en la mayor parte del contorno del implemento aparecen retoques secundarios por presión.
E) Sitio: Boulder 1 (Lámina 7, figs. E-E')

Material:(chert)

\section{Dimensiones:}

diámetro mayor: $55 \mathrm{~mm}$.

diámetro menor: $45 \mathrm{~mm}$.

espesor: $16.5 \mathrm{~mm}$.

La superficie convexa, conserva una porción de la corteza original, la superficie posterior corresponde al plano de percusión; junto al borde de contorno hay retoques a presión.

F) Sitio: Boulder 1 (Lámina 7, figs. F-F.')

Material: (chert)

\section{Dimensiones:}

longitud: $50 \mathrm{~mm}$

anchura: $41 \mathrm{~mm}$

espesor: $11 \mathrm{~mm}$.

Una de las superficies presenta parte de la corteza original; junto al borde semicircular anterior y lateral, el implemento presenta retoques por presión, la otra superficie aparece muy desbastada.

G) Sitio: Boulder 1 (Lámina 7, figs. G-G')

\section{Material: (chert)}

Dimensiones:

diámetro mayor: $47 \mathrm{~mm}$.

diámetro menor: $38 \mathrm{~mm}$.

espesor máximo: $16 \mathrm{~mm}$.

La superficie dorsal del artefacto está desbastada mediante percusión; en los bordes de contorno presenta dientes determinados por retoques hacia estos, la superficie posterior es el plano de percusión.

\section{H) Sitio: Boulder 1 (Lámina 7, figs. H-H')}

Material: (chert)

Dimensiones:

$$
\begin{aligned}
& \text { longitud: } 46 \mathrm{~mm} \text {. } \\
& \text { anchura: } 32 \mathrm{~mm} \text {. } \\
& \text { espesor: } 7 \mathrm{~mm} \text {. }
\end{aligned}
$$

La superficie dorsal fué trabajada mediante percusión bien controlada; la ventral, corresponde al plano de percusión, con bulbo de percusión muy visible. Hacia los bordes hay finos retoques por presión. Los tres bordes de utilización son aserrados. 


\section{Lascas Retocadas Rectangulares}

Entre la diversidad de lascas modificadas, aparecen dos formas bien definidas; las anteriormente descritas como triangulares, y otras más o menos rectangulares con un borde de utilización; algunas de la cuales describimos a continuación.

A) Sitio: Boulder (Lámina 8, figs. A-A')

\section{Material: (chert)}

Dimensiones:

longitud: $30.5 \mathrm{~mm}$.

anchura: $19.0 \mathrm{~mm}$.

espesor máximo: $10 \mathrm{~mm}$.

La superficie anterior está desbastada hacia el borde de utilización aserrado.

B) Sitio: Boulder 1 (Lámina 8, figs. B-B')

\section{Material: (chert)}

\section{Dimensiones:}

longitud: $24.5 \mathrm{~mm}$.

anchura máxima: $23.5 \mathrm{~mm}$.

espesor: $11.0 \mathrm{~mm}$.

La lasca está desbastada hacia el borde de utilización.

C) Sitio: Boulder 1 (Lámina 8, figs. C-C')

\section{Material: (chert)}

Dimensiones:

$$
\begin{aligned}
& \text { longitud: } 35 \mathrm{~mm} \text {. } \\
& \text { anchura: } 21.5 \mathrm{~mm} \text {. } \\
& \text { espesor: } 8 \mathrm{~mm} \text {. }
\end{aligned}
$$

Hacia el borde de utilización aserrado hay retoques por presión.

\section{Golpeadores Nucleares}

A) Sitio: Boulder 3 (Lámina 9, figs. A-A')

\section{Material: Diorita}

\section{Dimensiones:}

longitud: $125 \mathrm{~mm}$

anchura: $122 \mathrm{~mm}$.

espesor en la parte media: $575 \mathrm{~mm}$.

El artefacto de núcleo fue modificado mediante percusión directa; hay desbastamientos por uno de los lados, desde la parte media hasta el borde muy sinuoso; retoques por presión junto al borde, determinan aserramiento en el contorno.
B) Sitio: Boulder 1 (Lámina 9, figs. B-B')

\section{Material: (chert)}

\section{Dimensiones:}

longitud: $64 \mathrm{~mm}$.

anchura: $60 \mathrm{~mm}$.

espesor medio: $24 \mathrm{~mm}$.

El artefacto de núcleo está desbastado mediante percusión; junto al borde hay retoques por presión.

C) Sitio: Boulder 1 (Lámina 9, figs. C-C')

Material: (chert)

\section{Dimensiones:}

$$
\begin{aligned}
& \text { longitud: } 63.5 \mathrm{~mm} \text {. } \\
& \text { anchura: } 52 \mathrm{~mm} \text {. }
\end{aligned}
$$

espesor: $23.5 \mathrm{~mm}$.

Los desbastamientos por percusión en este artefacto de núcleo, se producen hacia uno de sus extremos, determinando un borde irregular.

\section{Raspadores Terminales}

Los raspadores terminales adoptan varias formas: la más significativa es la que a continuación describimos; en todos los raspadores terminales el material básico es chert, sólo se registró Diorita en un caso (A).

A) Sitio: Boulder 1

Material: Diorita

Dimensiones:

longitud: $68 \mathrm{~mm}$.

anchura máxima: $62.5 \mathrm{~mm}$.

espesor: $24 \mathrm{~mm}$.

\section{DISTRIBUCION PORCENTUAL DE LOS ELEMENTOS LITICOS OBTENIDOS EN LOS SITIOS I-II-III-IV DE BOULDER}

Lascas no modificadas

Fragmentos de núcleo

Raspadores discoidales

Lascas retocadas

Raspadores triangulares $\begin{array}{ccccc}\text { Boulder } & \text { Boulder } & \text { Boulder Boulder } & \text { Total } & \begin{array}{c}\text { Porcen- } \\ \text { taje }\end{array}\end{array}$

$\begin{array}{llllll}22 & 20 & 14 & - & 156 & 29.16\end{array}$

$\begin{array}{llllll}118 & 2 & 6 & 3 & 129 & 24.11\end{array}$


DISTRIBUCION PORCENTUAL DE LOS ELEMENTOS LITICOS OBTENIDOS EN LOS SITIOS I-II-III-IV DE BOULDER

Raspadores laterales Núcleos desbastados Raspadores cóncavos Raspadores terminales Golpeadores nucleares Fragmentos de metate Mano de moler

$\begin{array}{cccccc}\begin{array}{c}\text { Boulder } \\ 1\end{array} & \begin{array}{c}\text { Boulder } \\ \text { Boulder }\end{array} & 3 & \begin{array}{c}\text { Boulder } \\ \text { Total }\end{array} & \begin{array}{c}\text { Porcen- } \\ \text { taje }\end{array} \\ 26 & - & 6 & - & 32 & 5.98 \\ 14 & - & - & - & 14 & 2.62 \\ 10 & - & 2 & - & 12 & 2.24 \\ 5 & - & - & - & 5 & 0.93 \\ 1 & - & 1 & - & 2 & 0.37 \\ - & - & 2 & - & 2 & 0.37 \\ - & - & 1 & - & 1 & 0.19\end{array}$

BOULDER I- II- III - IV

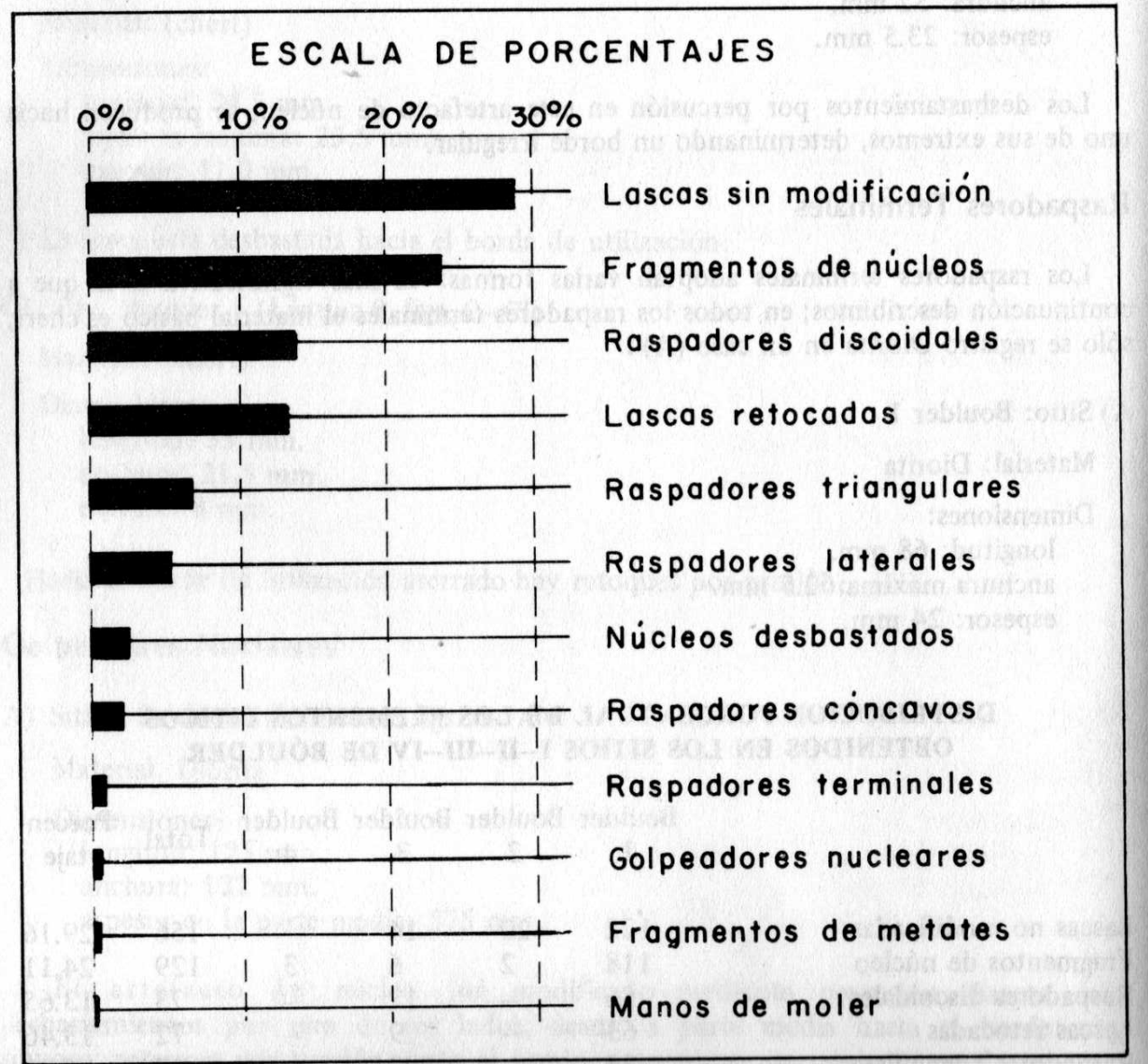

\section{CONSIDERACIONES FINALES}

Como queda anotado, el mayor número de elementos líticos obtenidos en la recolección superficial de los sitios de Boulder, corresponde a instrumentos de lasca monofaciales.

Dentro de los elementos obtenidos, el mayor valor porcentual corresponde a la categoría de raspadores (discoidales, triangulares, laterales, cóncavos, terminales), cuyo porcentaje cubre el $29.720 / 0$ de los elementos obtenidos; también es alta la frecuencia de lascas sin modificar y fragmentos de núcleo. Este hecho ya sugiere que en alguna época los sitios de Boulder constituyeron la estación de grupos trashumantes; aunque no fué posible la obtención de restos de fauna en asociación con los elementos líticos, el utillaje recolectado indica una subsistencia fundamental a base de caza y recolección.

El material básico en la elaboración de los implementos es chert, abundante en los rodados de las quebradas de la región, pero excepcionalmente aparecen entre los implementos algunos cuyo material básico es diorita, andesita o en un caso cuarzo estos últimos materiales tuvieron que transportarse.

La técnica mas frecuentemente utilizada es la de percusión, pero en un gran número de los elementos son observables retoques secundarios marginales obtenidos mediante presión, característica que anotamos al hacer la descripción de algunos artefactos.

La presencia de fragmentos de metate y mano de moler entre los elementos obtenidos en el sitio 1 (Boulder 1), no descarta la posibilidad de un temprano arcaico, aunque las características de los implementos, la ausencia total de cerámica y la situación inmediatamente sobre los estratos de la formación Terraza de Aipe, conduce a considerar la posibilidad de que las industrias aquí obtenidas se remonten a una etapa anterior.

Aunque la vaga frecuencia de los materiales obtenidos no permite establecer conclusiones, menos cuando carecemos de elementos cronológicos, como particularidad especial en los elementos líticos obtenidos en Boulder, encontramos estrecha similitud con los hallados en otros sitios precerámicos de Colombia; tanto la técnica de elaboración como las características morfológicas de los implementos de Boulder recuerdan algunos elementos del Abra (Zipaquirá, Cundinamarca) (ver Correal, Van-der-Hammen y Lerman, 1970). Tal por ejemplo, los artefactos muy crudos de lasca monofaciales y algunos tipos de raspadores. Otros elementos, lascas en su mayoría, recuerdan las industrias de la Costa Atlántica de Colombia (ver Reichel-Dolmatoff, 1965) y otros en fin recuerdan elementos obtenidos en el Magdalena Medio (como el Clavo-Puerto Carare); entre estos muy característico el Chopper; es evidente que a través de la vía natural del río Magdalena se verificaran desde hace más de 10 milenios desplazamientos; la fecha más antigua hasta ahora obtenida para las industrias líticas corresponde al Abra, Cundinamarca $12.400 \pm 160$ B.P. $y$ en excavaciones efectuadas en la región del Tequendama (Sabana de Bogotá) pudimos obtener una cronología de 9.700 B.P. para estratos con elementos líticos similares a algunos de Boulder; en este orden de ideas es posible que los elementos líticos aquí presentados puedan remontarse al propio comienzo del tardi-glacial 


\section{BIBLIOGRAFIA}

Burgl, H. "Artefactos paleolíticos de una tumba en Garzón (Huila)". Revista Colombiana de Antropologla Vol. VI. Bogotá, 1957.

Correal, G.; Van-Der-Hammen, T. y Lerman L.- "Artefactos Líticos de Abrigos Rocosos en al Abra, Colombia." Revista Colombiana de Antropología. Vol. XIV. 1966-1969

Reichel-Dolmatoff, G.- Excavaciones Arqueológicas en Puerto Hormiga. Departamento de Bollvar Antropología 2. Edi. Univ. de los Andes, Bogotá, 1965.

Van-Der-Hammen T. "Las terrazas del río Magdalena y la posición estratigráfica de los hallazgos de Garzón." Revista Colombiana de Antropología Vol. VI. Bogotá, 1957.

Vogel, J.C. \& Lerman, L.- "Groningen. Radiocarbon dates VII." Radiocarbon Vol II 2. 1969.

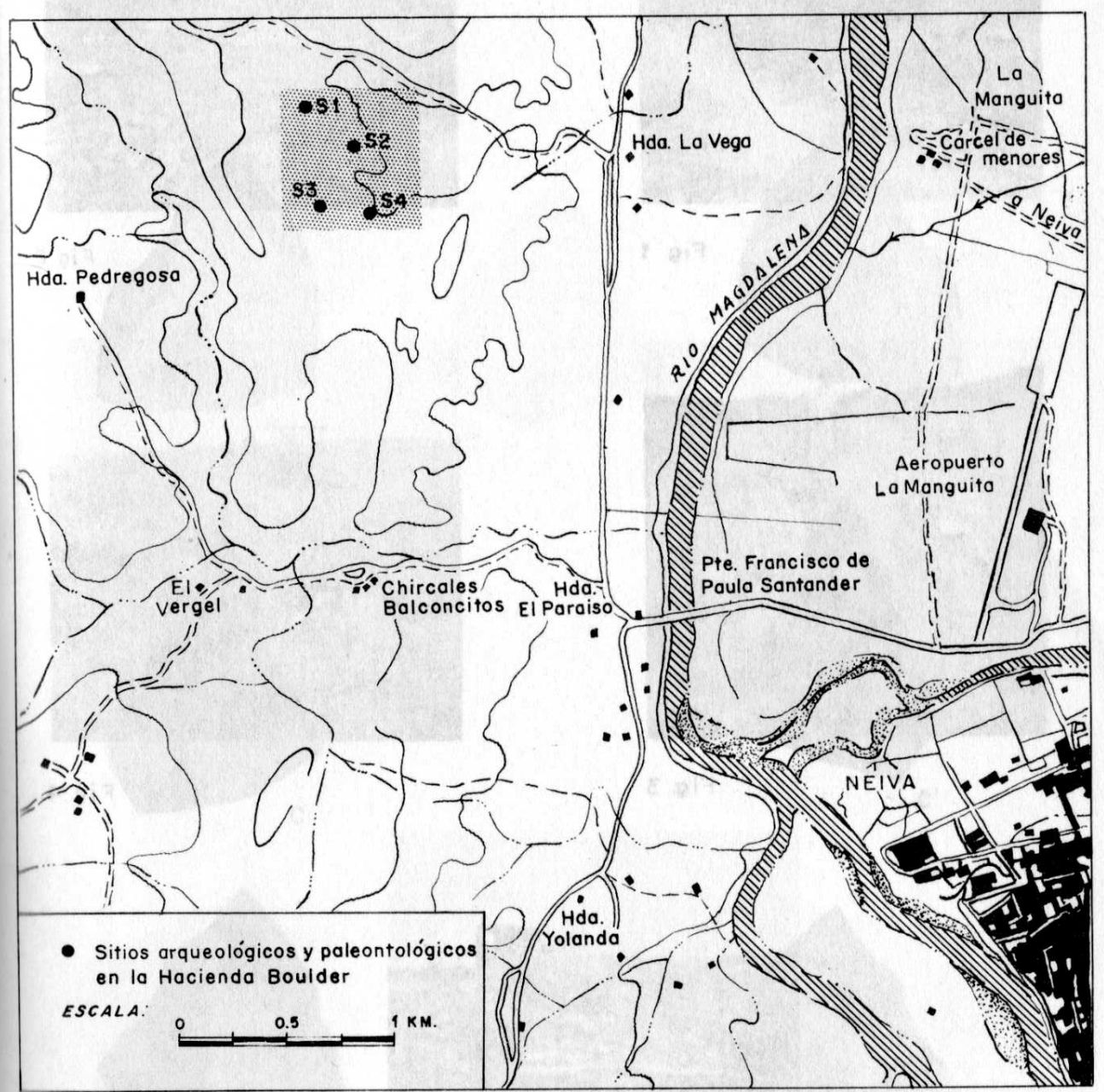




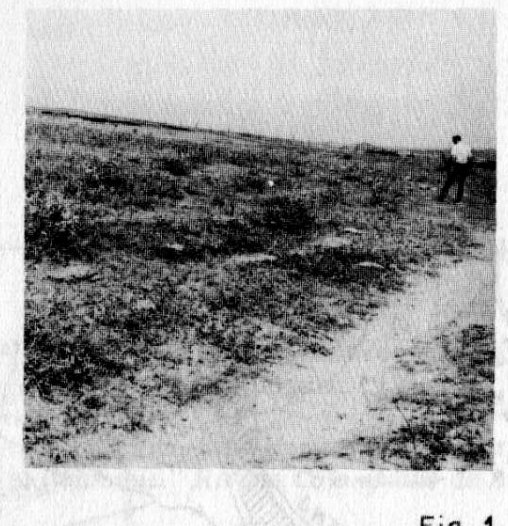

Fig. 1

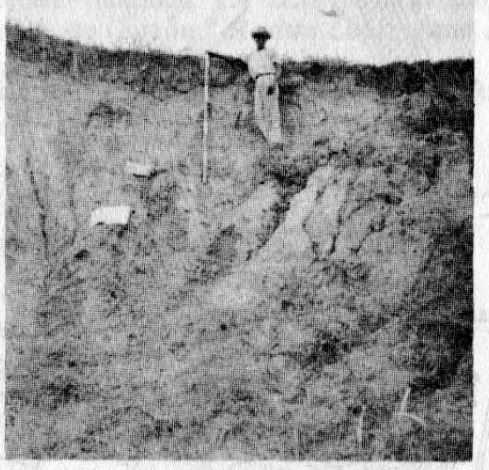

Fig. 3

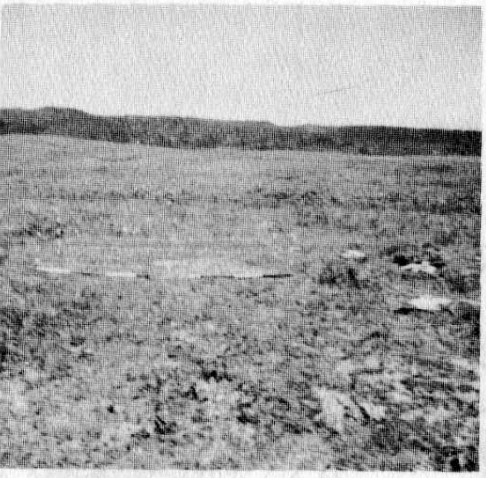

Fig. 2

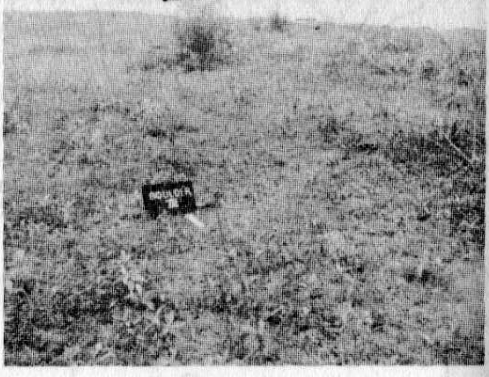

Fig. 4

is ant

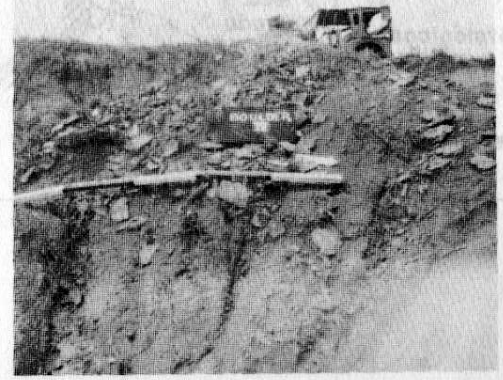

Lámina 1
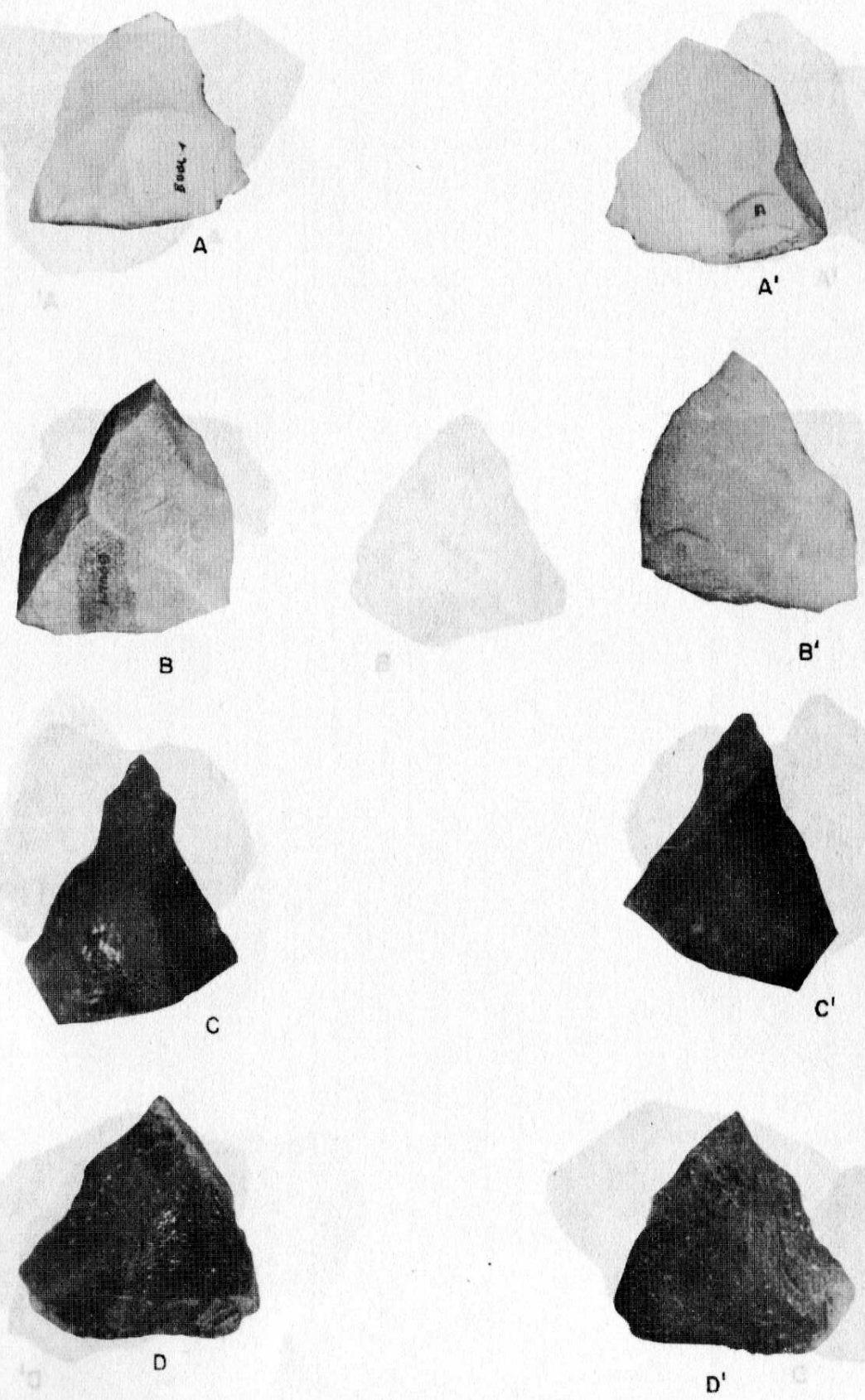

Lamina 2 


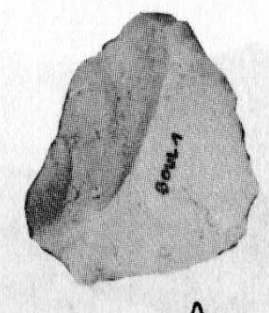

A
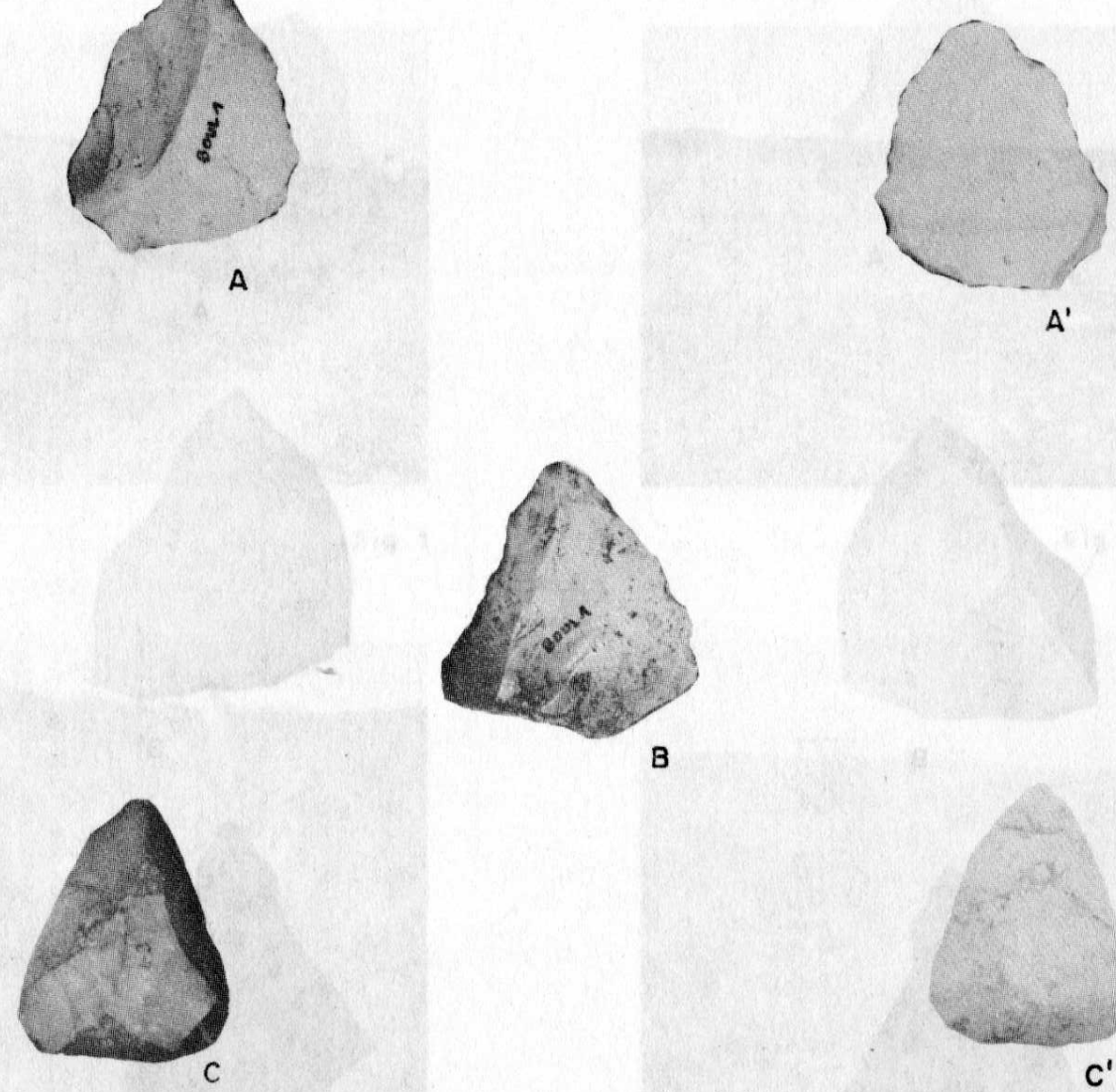

B
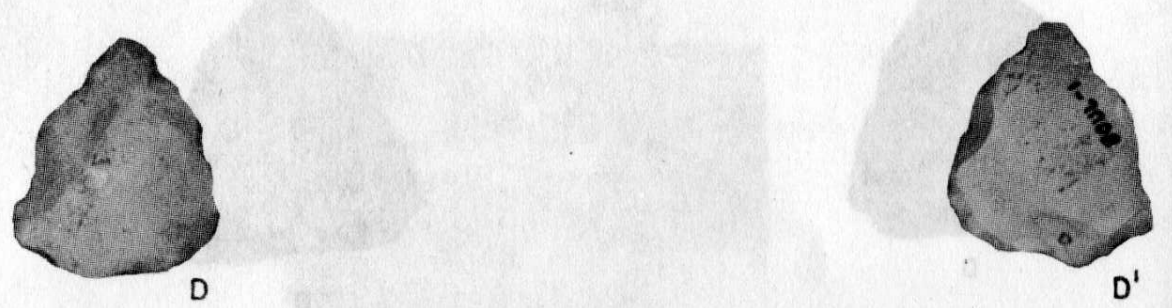

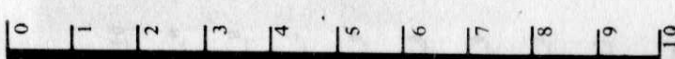
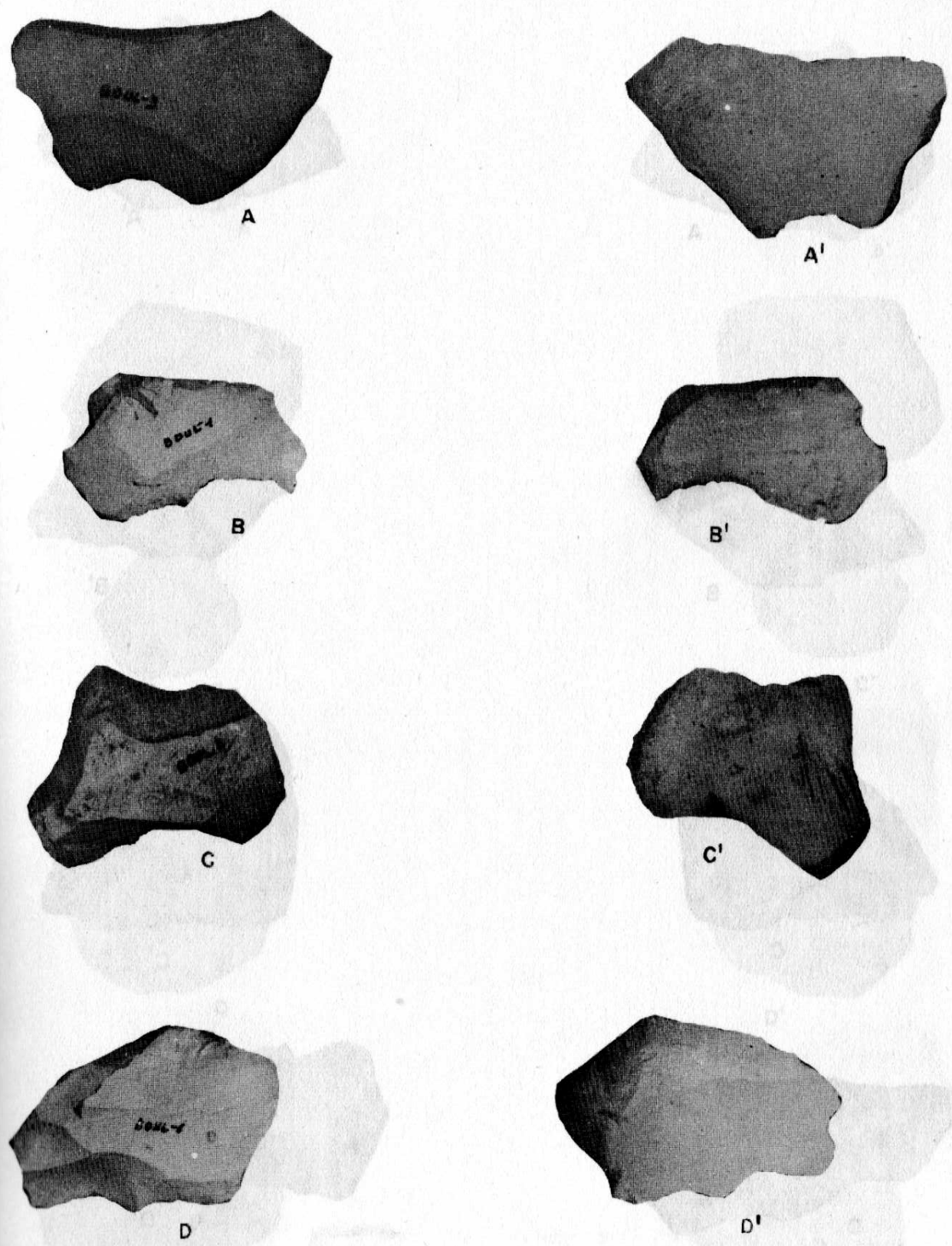

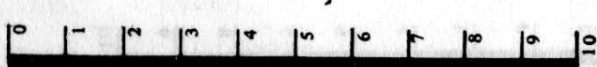

Lómina 4 

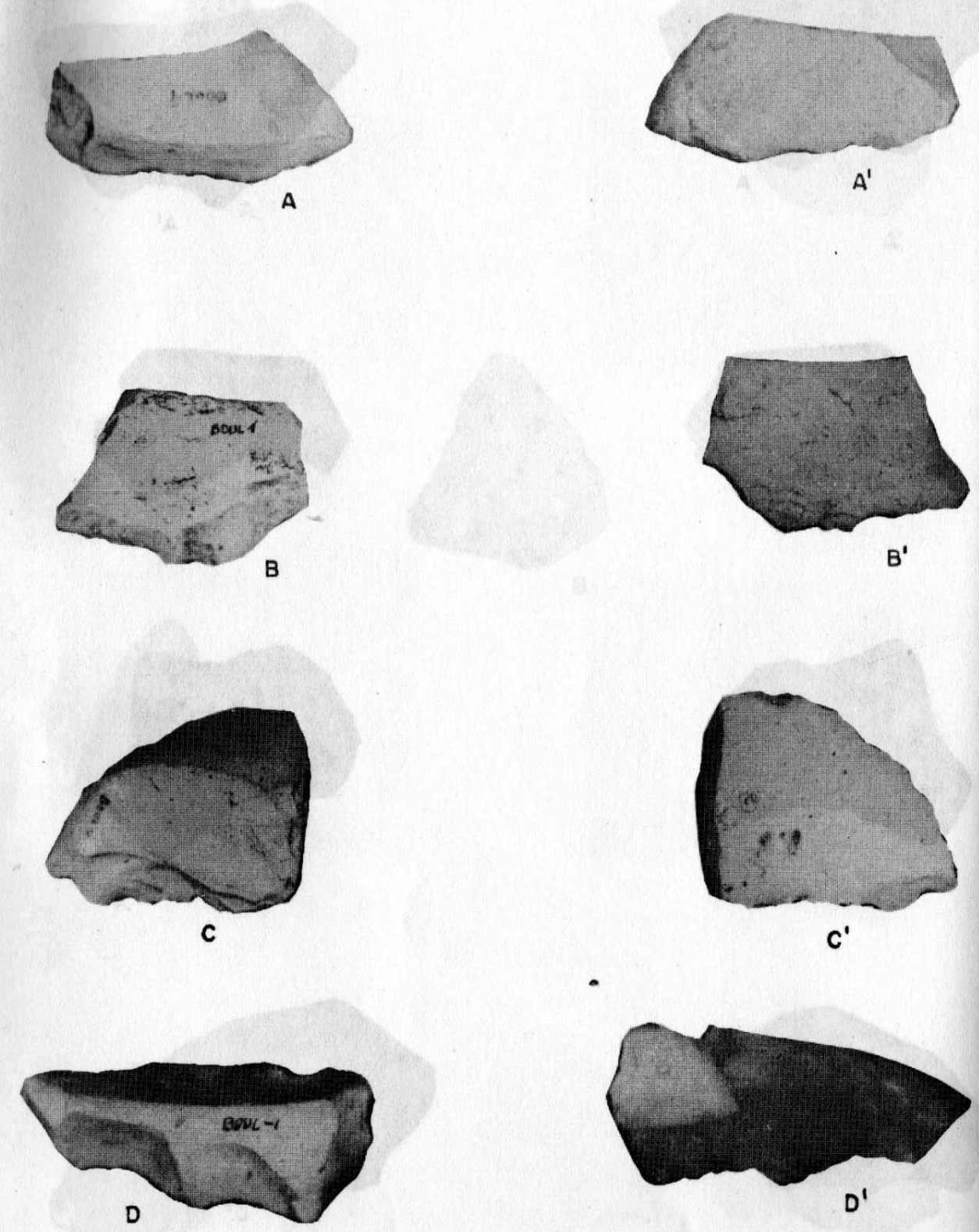

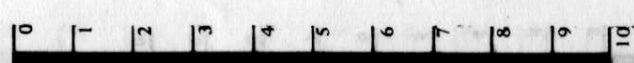
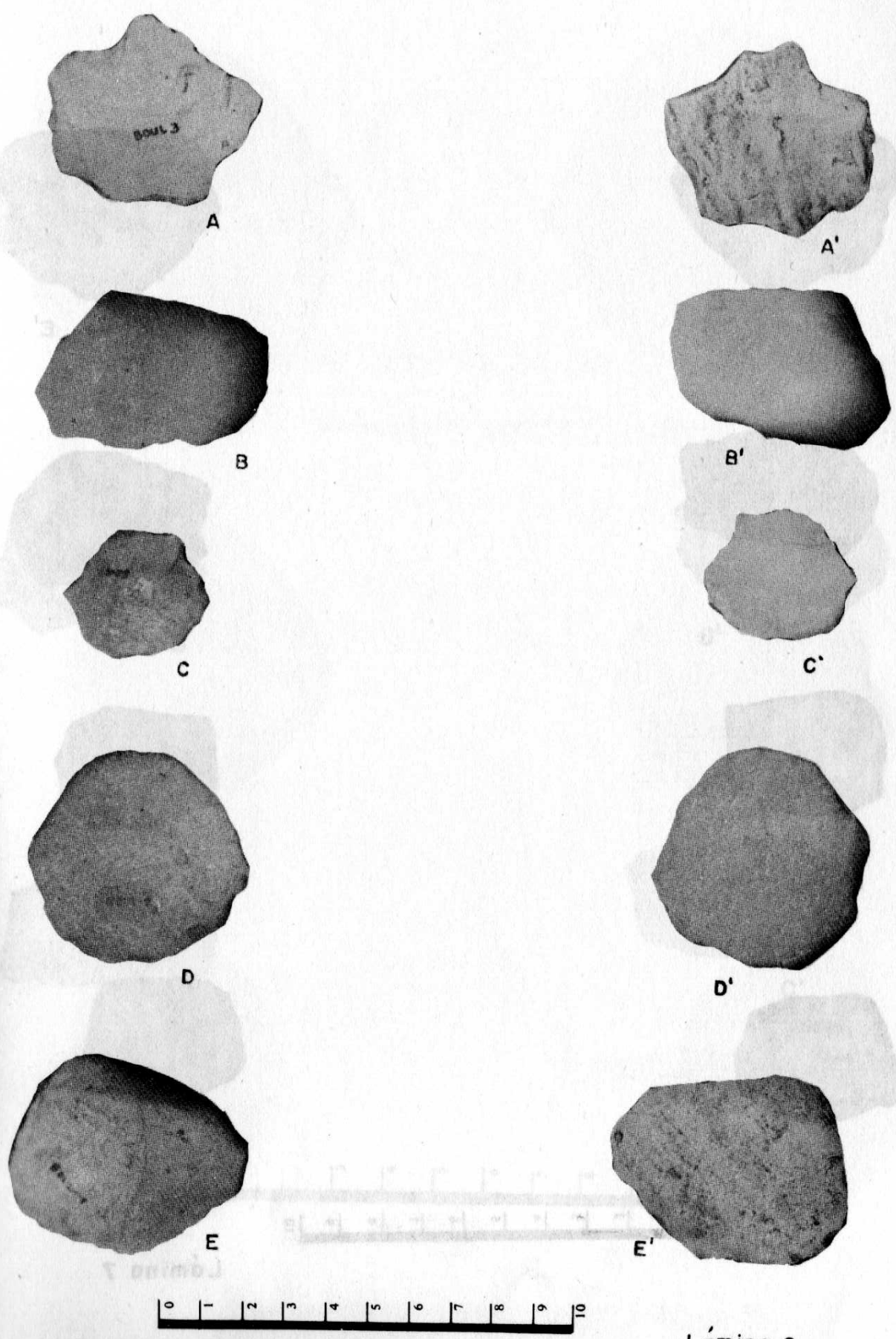

Lámina 6 

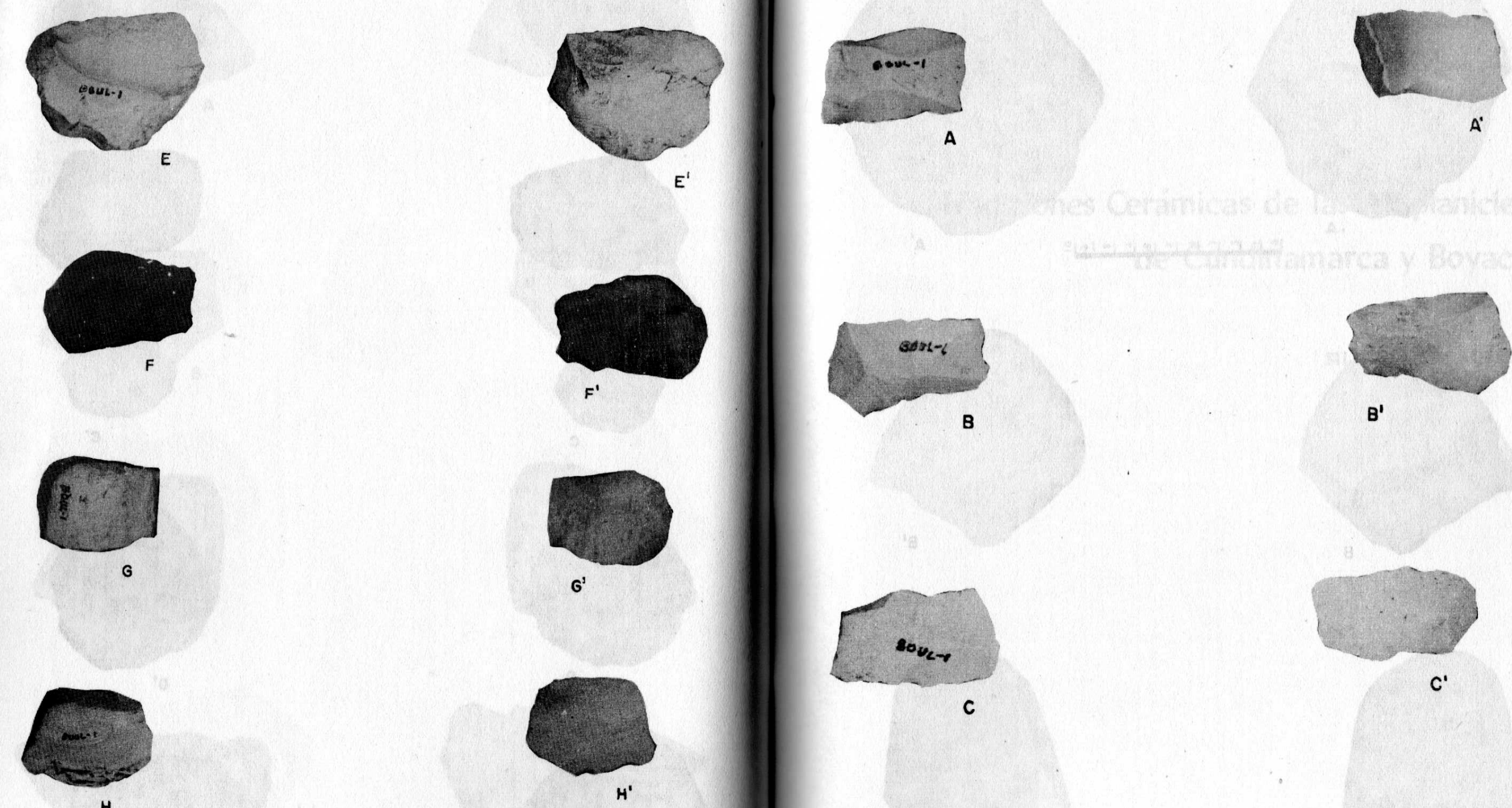

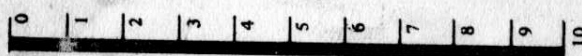




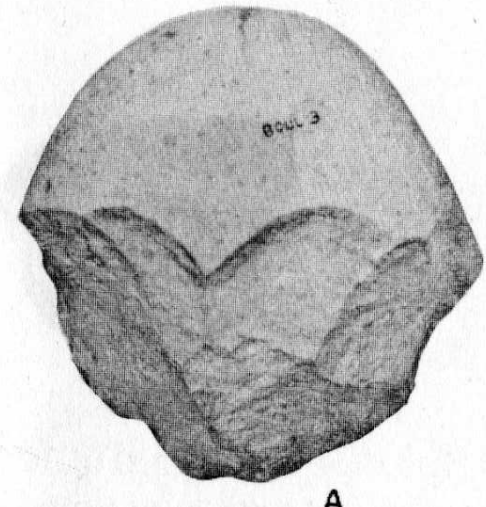

A
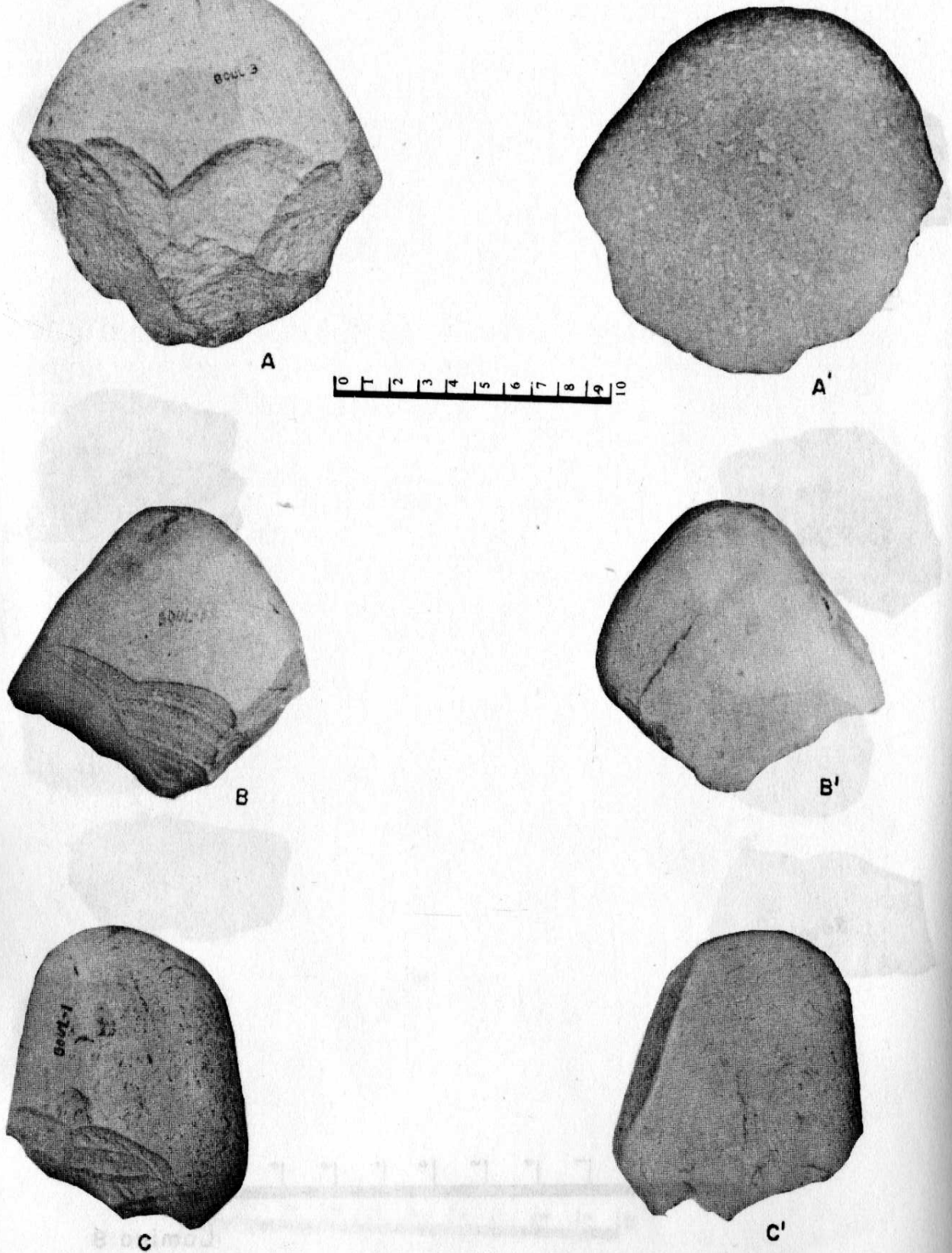

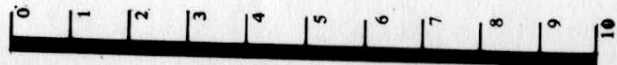

Lómina 9 